\title{
Analysis of the Risk Factors for Intraoperative Acute Diffuse Brain Swelling in Patients With Isolated Traumatic Acute Subdural Haematomas
}

\section{Ruhong Wu}

The Third Affiliated Hospital of Soochow University

Chunbo Liu

The Third Affiliated Hospital of Soochow University

Tao Ma

The Third Affiliated Hospital of Soochow University

Geng Jia

The Third Affiliated Hospital of Soochow University

Huaping Qin ( $\nabla$ qhp197708@163.com )

The Third Affiliated Hospital of Soochow University

\section{Research Article}

Keywords: Acute subdural hematoma, acute diffuse brain swelling, decompressive craniotomy

Posted Date: February 15th, 2022

DOI: https://doi.org/10.21203/rs.3.rs-1256746/v1

License: (9) This work is licensed under a Creative Commons Attribution 4.0 International License. Read Full License 


\section{Abstract}

Background: The purpose of this retrospective study was to investigate the risk factors for intraoperative acute diffuse brain swelling in patients with isolated traumatic acute subdural haematomas (ASDH).

Methods: A total of 256 patients who underwent decompressive craniotomy for isolated traumatic ASDH between April 2013 and December 2020 were included. We evaluated the risk factors for intraoperative acute diffuse brain swelling using a multivariate logistic regression analysis.

Results: The incidence of intraoperative acute diffuse brain swelling in patients with isolated traumatic ASDH was 21.88\% (56/256). Dilated pupils (OR=24.78), subarachnoid haemorrhage $(O R=2.41)$, and the time from injury to surgery $(\mathrm{OR}=0.32)$ were independent predictors of the risk factors for intraoperative acute diffuse brain swelling, while no independent associations were observed between the risk factors and sex, age, the Glasgow Coma Scale score, site of haematoma, thickness of haematoma, midline shift and the status of the basal cistern, although the Glasgow Coma Scale score and the status of the basal cistern were correlated with the incidence of intraoperative acute diffuse brain swelling in the univariate analyses.

Conclusions: This study identified the risk factors for intraoperative acute diffuse brain swelling in patients with isolated traumatic ASDH. An increased risk of intraoperative acute diffuse brain swelling occurs in patients with bilaterally dilated pupils, subarachnoid haemorrhage and a shorter time from injury to surgery. These findings should help neurosurgeons obtain information before surgery about the intraoperative acute diffuse brain swelling in patients with isolated traumatic ASDH.

\section{Background}

Acute subdural haematoma (ASDH) is a common and serious injury in traumatic brain injury (TBI) patients. Decompressive craniotomy (DC) is recommended when patients have a decreased level of consciousness, a greater size of the haematoma, a midline shift, or a basal cistern obliteration.[1] Acute encephalocele is a very dangerous and urgent situation during DC. The main causes of acute encephalocele include a contralateral subdural haematoma, a contralateral epidural haematoma and acute diffuse brain swelling.[2,3] Acute diffuse brain swelling is one of the leading causes of intraoperative acute encephalocele, and it also has the highest mortality. Neurosurgeons can deal with remote intracranial haematomas by reoperation, but are often caught off guard because there is no effective method for diffuse brain swelling. Acute diffuse brain swelling during a DC is often accompanied by an acute drop in blood pressure, and the mortality is very high. Our neurosurgeons are very afraid of encountering this situation. However, few reports have evaluated the risk factors for intraoperative acute diffuse brain swelling in patients with isolated traumatic ASDH. This information is urgently needed in clinical work to estimate the incidence of intraoperative acute diffuse brain swelling based on preoperative clinical and imaging data, and this information can help neurosurgeons accurately determine the surgical risk and to provide reasonable suggestions to patients' families. In this study, we 
aimed to explore the risk factors for intraoperative acute diffuse brain swelling in patients with isolated traumatic ASDH.

\section{Methods}

\section{Patient Population}

This retrospective study was performed on 256 patients who underwent surgery after being diagnosed with isolated traumatic ASDH at our neurosurgical department between April 2013 and December 2020. We set the inclusion and exclusion criteria for this retrospective study, with the final number of cases decided according to the inclusion and exclusion criteria. The inclusion criteria for this retrospective study were as follows: 1) the ASDH caused by trauma, 2) the ASDH located on the convex surface of the brain, 3 ) the patient had an emergency DC that was performed immediately after admission, and 4) the patient's age was between 18-80 years old. The following exclusion criteria were applied: 1) patients with penetrating head injuries, acute epidural haematomas, ASDH of the posterior fossa, intracerebral haematomas and serious extracranial injuries; 2 ) patients whose time from injury to surgery exceeded 8 hours; 3) patients whose preoperative blood pressure was lower than $90 / 60 \mathrm{mmHg}$; 4) patients who take anticoagulants or antiplatelet drugs; and 5) patients who had severe diseases of the heart, lungs, liver, kidneys, or haematologic system. Age, sex, pupil reaction, site of haematoma, thickness of haematoma, midline shift, subarachnoid haemorrhage (SAH), status of the basal cistern and time from trauma to surgery were investigated. The study was approved by the Ethics Committee of the Third Affiliated Hospital of Soochow University.

\section{General patient management}

According to the above inclusion and exclusion criteria, we finally selected and analysed 256 patients. We categorized all of the variables into three groups if the variables might be related to the risks of intraoperative acute diffuse brain swelling of isolated traumatic ASDH: 1) the clinical variables included sex, age, the Glasgow Coma Scale (GCS) score, and pupillary reaction; 2) the computerized tomography (CT) variables included the site of haematoma, midline shift, thickness of the haematoma, the status of the basal cistern, and SAH; and 3) the surgical variable was the time from trauma to surgery.

After an isolated traumatic ASDH was diagnosed and when there were indications for surgery, each patient underwent a $D C$ through a question mark incision. First, a $5 \mathrm{~cm}$ incision was made in the temporal area along the question mark line, a hole was drilled into the skull, a small hole was cut in the dura to try to slowly release the liquid ASDH, and then a DC was performed. The size of the skull bone flap was approximately $10 \mathrm{~cm}$ by $10 \mathrm{~cm}$. The dura was opened, and the haematoma was evacuated in all of the patients. The criteria for intraoperative acute diffuse brain swelling were as follows: after opening the dura, an encephalocele immediately appeared, the texture of the brain tissue was hard, and there was almost no obvious brain pulsation. At the same time, the acute encephalocele may be accompanied by a progressive decrease in the patient's blood pressure. Blood transfusions and volume expansion could not 
correct the patient's hypotension. After emergency closing of the cranial cavity, a brain CT scan was then immediately performed and did not find new haematomas.

\section{Clinical variables}

The GCS scores of the patients were determined on admission, and all patients were divided into three categories $(3-5,6-8,9-15)$. The preoperative pupillary reaction was divided into two categories: no dilated pupils and unilaterally dilated, bilaterally dilated pupils.

\section{CT variables}

The maximum thickness ( $<5 \mathrm{~mm}, 5-9.9 \mathrm{~mm}, 10-14.9 \mathrm{~mm}, \geq 15 \mathrm{~mm}$ ) and midline shift at the septum pellucidum ( $<5 \mathrm{~mm}, 5-9.9 \mathrm{~mm}, 10-14.9 \mathrm{~mm}, \geq 15 \mathrm{~mm}$ ) were analysed according to preoperative CT scans. The condition of the basal cistern was evaluated using the axial image at the level of the midbrain, which was then divided into 3 branches (one posterior and two laterally) (Figure 1). Each branch can be assessed separately as to whether or not it is open or occluded. For the logistic regression analysis, patients were divided into four categories $(0,1,2,3$ branches occluded).

\section{Surgical variables}

The American College of Surgeons Committee on Trauma set a demarcation line for whether patients with traumatic ASDH undergo surgery within 4 hours.[4] According to this criteria, we divided all patients into two categories ( $<4$ hours, $\geq 4$ hours) according to the time from trauma to surgery.

\section{Statistical analysis}

The statistical analyses of the data were carried out using SPSS 22.0 software IBM Corp., Armonk, New York, USA). Discrete variables were compared using the chi-square test, and continuous variables were compared using the Mann-Whitney $U$ test. All variables were used as candidates and put into a multivariate logistic regression model to identify which variables were independently associated with the risk factors for intraoperative acute diffuse brain swelling in patients with isolated traumatic ASDH, and all variables with $\mathrm{P}<0.05$ were considered to have statistical significance.

\section{Results}

\section{Univariate analysis of the risk factors for intraoperative acute diffuse brain swelling}

Of the 256 patients, 56 patients experienced intraoperative acute diffuse brain swelling, and the overall incidence was $21.8 \%$. The median age of these patients was 57 years (range $18-79$ years). Male patients constituted $72.3 \%$ of the study population, which included 185 men and 71 women. Ten variables contributing to intraoperative acute diffuse brain swelling were analysed separately in the univariate analyses. Consequently, five factors were found to be significantly related to intraoperative acute diffuse brain swelling (Table 1). 
Table 1. Variables related to the risk factors for intraoperative acute diffuse brain swelling in patients with isolated traumatic ASDH $(n=256)$

\begin{tabular}{|c|c|c|c|c|}
\hline $\begin{array}{r}\mathrm{Nc} \\
\text { diffu } \\
\text { swe. }\end{array}$ & $\begin{array}{l}\text { acute } \\
\text { ase brain } \\
\text { ling }\end{array}$ & $\begin{array}{l}\text { Acute } \\
\text { diffuse brain } \\
\text { swelling }\end{array}$ & Statistical values & $p$ value \\
\hline Totals & 200 & $56(21.8 \%)$ & & \\
\hline Sex & & & 0.731 & 0.393 \\
\hline Male & 142 & $43(23.2 \%)$ & & \\
\hline Female & 58 & $13(18.3 \%)$ & & \\
\hline Age (years) & 57.5 & 55.5 & 5399 & $0.681^{*}$ \\
\hline GCS & & & 35.198 & $<0.001$ \\
\hline $3-5$ & 121 & $55(31.3 \%)$ & & \\
\hline $6-8$ & 64 & $1(1.5 \%)$ & & \\
\hline $9-15$ & 15 & $0(0 \%)$ & & \\
\hline Dilated pupils & & & 52.332 & $<0.001$ \\
\hline Absent or Unilateral & 120 & $3(2.4 \%)$ & & \\
\hline Bilateral & 80 & $53(39.8 \%)$ & & \\
\hline Site of haematoma & & & 0.333 & 0.56 \\
\hline Left & 98 & $25(20.3 \%)$ & & \\
\hline Right & 102 & $31(23.3 \%)$ & & \\
\hline Thickness of haemato & $\mathrm{ma}(\mathrm{mm})$ & & 4.303 & 0.231 \\
\hline$<5$ & 10 & $4(28.6 \%)$ & & \\
\hline $5-9.9$ & 87 & $16(15.5 \%)$ & & \\
\hline $10-14.9$ & 72 & $26(26.5 \%)$ & & \\
\hline$\geqslant 15$ & 31 & $10(24.4 \%)$ & & \\
\hline Midline shift (mm) & & & 2.170 & 0.538 \\
\hline$<5$ & 8 & $4(33.3 \%)$ & & \\
\hline $5-9.9$ & 52 & $13(20.0 \%)$ & & \\
\hline $10-14.9$ & 89 & $21(19.1 \%)$ & & \\
\hline$\geqslant 15$ & 51 & $18(26.1)$ & & \\
\hline $\mathrm{SAH}$ & & & 9.131 & 0.003 \\
\hline No & 117 & $20(14.6 \%)$ & & \\
\hline Yes & 83 & $36(30.3 \%)$ & & \\
\hline \multicolumn{3}{|c|}{ Condition of the basal cistern } & 38.450 & $<0.001$ \\
\hline 0 & 9 & $0(0.0 \%)$ & & \\
\hline 1 & 30 & $0(0.0 \%)$ & & \\
\hline 2 & 83 & $9(9.8 \%)$ & & \\
\hline 3 & 78 & $47(37.6 \%)$ & & \\
\hline \multicolumn{3}{|c|}{ The time from injury to surgery (h) } & 11.979 & 0.001 \\
\hline$<4$ & 122 & $48(28.2 \%)$ & & \\
\hline$\geq 4$ & 78 & $8(9.3 \%)$ & & \\
\hline
\end{tabular}

GCS, Glasgow Coma Scale; SAH, subarachnoid haemorrhage.

The incidence of intraoperative acute diffuse brain swelling had no significant correlation with sex or age $(p>0.05)$. The incidence of intraoperative acute diffuse brain swelling had a significant correlation with 
the GCS score $(p<0.05)$. Of the 176 patients with GCS scores ranging from 3 to $5,55(31.1 \%)$ experienced intraoperative acute diffuse brain swelling. In contrast, only 1 (1.5\%) of the 65 patients with GCS scores ranging from 6 to 8 experienced intraoperative acute diffuse brain swelling, and no patients with GCS scores ranging from 9 to 15 experienced intraoperative acute diffuse brain swelling. The incidence of intraoperative acute diffuse brain swelling had a significant correlation with dilated pupils $(p<0.05)$. Of the 133 patients with bilaterally dilated pupils, 53 (39.8\%) experienced intraoperative acute diffuse brain swelling. In contrast, intraoperative acute diffuse brain swelling occurred in only $3(2.4 \%)$ of the 123 patients, with no dilated pupils or unilateral pupil dilation.

The incidence of intraoperative acute diffuse brain swelling had no significant correlation with the site of haematoma, the thickness of haematoma or midline shift $(p>0.05)$. The incidence of intraoperative acute diffuse brain swelling had a significant correlation with SAH $(p<0.05)$. Of the 119 patients with SAH, 36 (30.3\%) experienced intraoperative acute diffuse brain swelling. In contrast, acute diffuse brain swelling occurred in only $24(14.6 \%)$ of the 137 patients without traumatic SAH.

The condition of the basal cistern was significantly correlated with the incidence of intraoperative acute diffuse brain swelling $(p<0.05)$. The incidence $(37.6 \%)$ of intraoperative acute diffuse brain swelling in patients with 3 branches occluded was significantly higher than that in the other 3 groups. The time from injury to surgery significantly influenced the incidence of intraoperative acute diffuse brain swelling $(p<0.05)$. The incidence of intraoperative acute diffuse brain swelling $(28.2 \%)$ in patients undergoing surgery within 4 hours after injury was higher than that in the other patients $(p=0.001)$.

\section{Multivariate analysis of risk factors for acute diffuse brain swelling}

The multivariate logistic regression analysis showed that dilated pupils, $\mathrm{SAH}$, and the time from injury to surgery were independent predictors of the risk factors for intraoperative acute diffuse brain swelling in patients with isolated traumatic ASDH. The odds ratios (ORs) of these three variables and the regression formulas are summarized in Table 2. No independent association was observed between the incidence of intraoperative acute diffuse brain swelling and age, sex, the GCS score, the site of haematoma, the thickness of haematoma, midline shift or the condition of the basal cistern.

Table 2. Independent predictors of the risk factors for intraoperative acute diffuse brain swelling in patients with isolated traumatic ASDH by multivariate logistic analysis. 


\begin{tabular}{lcc}
\hline Factors & OR $(95 \% \mathrm{CI})$ & $p$ value \\
\hline Dilated pupils & & \\
Absent or Unilateral & 1.00 & \\
Bilateral & $24.78(7.39-83.09)$ & 0.000 \\
SAH & 1.00 & \\
$\quad$ No & $2.40(1.19-4.84)$ & 0.014 \\
$\quad$ Yes & 1.00 & \\
The time from injury to surgery & $0.32(0.13-0.77)$ & 0.011 \\
$\quad<4$ & \\
$\geq 4$ &
\end{tabular}

SAH, subarachnoid haemorrhage.

Cl: confidence interval OR: odds ratio

\section{Discussion}

Traumatic ASDH is the most common condition in severe head trauma patients. It occurs in approximately $30 \%$ of patients with severe head injury and has reported mortality rates up to $60 \%$ for patients treated within the last decade.[5-7] It leads to serious public health, social, and economic concerns. For traumatic ASDH with surgical indications, DC is the most commonly used surgical technique.[8]

Previous reports have shown that an acute encephalocele will significantly increase the mortality of patients with acute ASDH.[9] Almost every neurosurgeon will experience it. The common causes include contralateral subdural or epidural haematomas, epidural haematomas in the confluence of sinus areas, acute traumatic cerebral infarctions, and acute diffuse brain swelling.[10] In these cases, acute diffuse brain swelling is particularly dangerous, with a high mortality and a poor prognosis.[9] How to judge the risk of intraoperative acute diffuse brain swelling in patients with traumatic ASDH based on the preoperative clinical data and imaging data is a problem that our neurosurgeon is more concerned about because this information can help us more accurately determine the operation risks and give the patients' families more accurate advice.

Acute diffuse brain swelling usually occurs rapidly after the dura is opened, which is different from acute epidural haematomas or acute subdural haematomas in remote sites because these usually occur after a period of time after the dura is opened. The cerebral vein is in a state of obvious congestion with a dark purple colouring, and there may be accompanying cerebral venous rupture and haemorrhage. The texture of the brain tissue becomes hard without brain pulsation, and brain tissue "fermentation"-like bulges are usually accompanied by a sharp drop in blood pressure, while blood transfusions or fluid rehydration often cannot maintain the patient's blood pressure. The prognosis is very poor, with most patients dying within 7 days after the operation. The main mechanism of intraoperative acute diffuse brain swelling is the external force causes damage to the cerebrovascular motor centre after brain injury, which in turn 
causes the loss of cerebrovascular autoregulation. Then, the brain tissue becomes congested, and the cerebral blood flow and blood volume increase rapidly,[11] which leads to acute diffuse brain swelling. When the dura is opened during DC, the intracranial pressure (ICP) drops rapidly, and the cerebral blood vessels become acutely dilated. During this time, the brain tissue is in a hyperperfusion condition, and the cerebral vascular tension centre is damaged, $[12,13]$ which makes it difficult to retract the blood vessels. Then, diffuse brain swelling occurs.

We observed that the incidence of intraoperative acute diffuse brain swelling was $21.8 \%$ in 256 surgical patients with isolated traumatic ASDH. The multivariate logistic regression analysis indicated that dilated pupils, $\mathrm{SAH}$, and the time from injury to surgery were independently associated with the incidence of acute diffuse brain swelling.

In 256 surgical patients with traumatic ASDH, the incidence of intraoperative acute diffuse brain swelling in patients with bilaterally dilated pupils was significantly higher than that of patients without dilated pupils and patients with unilateral dilated pupils. TBI can cause rupture of the cerebral arteries and veins and swelling of the cerebral hemispheres. Due to the space-occupying effect of the subdural haematoma and the swollen brain tissue, the ICP is significantly increased, resulting in obstruction of venous return and cerebral oedema, which in turn increase the ICP, causing brain herniation. Cerebral herniation leads to a severe cerebral venous return disorder, which forms a vicious circle. This is closely related to the theory of the pathogenesis of intraoperative acute diffuse brain swelling.[13,14] Dilated pupils in patients with TBI not only indicate that the patient has herniated or is in the preherniation stage but also indicate that the prognosis is poor. According to some scholars, the mortality of patients with bilateral dilated pupils is as high as $64 \%-88.1 \% .[15,16]$ Bilaterally dilated pupils indicate that the patient's brainstem has been more severely compressed, the patient's brain swelling is more serious, and these findings are more likely to cause intraoperative acute diffuse brain swelling.

Although the multivariate logistic regression analysis indicated that the GCS score was not independently associated with the incidence, the univariate analysis indicated that the GCS score was highly correlated with the incidence of acute diffuse brain swelling during surgery. Patients with lower GCS scores had a higher incidence of intraoperative acute diffuse brain swelling than patients with higher GCS scores. A GCS score lower than 5 illustrates that the patient's primary brain injury is severe, and the shear stress generated by rotating external force damages the vascular motor centres, resulting in the loss of cerebrovascular autoregulation and acute cerebrovascular expansion. The increase in the cerebral blood volume leads to brain swelling, which can easily lead to intraoperative acute diffuse brain swelling. Patients with dilated pupils tend to have lower GCS scores than the patients with nondilated pupils. It is recognized that the state of the patient's pupil is closely related to the prognosis. According to previous reports, the mortality is $100 \%$ in patients with a GCS score of 3 points combined with bilateral pupil dilation.[17] Analysis of the prognostic factors in ASDH patients found that the GCS score is positively correlated with the prognosis of patients with ASDH, but it has not been proven to reflect the level of the brainstem injury.[18, 19] Notably, evaluation of the GCS score is usually hindered by the presence of longlasting sedatives and paralytics. The evolution of the clinical status cannot be detected in patients who 
are paralyzed pharmacologically with long-acting agents. Therefore, the GCS score of the patient at admission may be lower than the patient's true GCS score.

The multivariate logistic regression analysis showed that SAH was an independent risk factor for intraoperative acute diffuse brain swelling in patients with traumatic ASDH. Blood cells in the subarachnoid space can release substances that cause vasospasm, which can cause spasm of the cerebral arteries, and can cause intracranial hypertension, cerebral oedema and even cerebral herniation, [20] which may be why patients with SAH are prone to intraoperative acute diffuse brain swelling. The presence of SAH has been shown to be a strong predictor of both the outcome and mortality in TBI patients.[16, 21-23]

The multivariate logistic regression analysis indicated that the condition of the basal cistern was not independently associated with the incidence of intraoperative acute diffuse brain swelling, but the univariate analysis indicated that the incidence of intraoperative acute diffuse brain swelling in patients with 3 branches occluded was significantly higher than the incidence in the other 3 groups. The degree of compression of the basal cistern reflects not only the degree of ICP but also reflects the degree of brainstem compression. The occluded basal cistern indicates that the supratentorial brain tissue compresses the brainstem, which is an important prognostic factor that is associated with a poor outcome and death. The occluded basal cistern is often accompanied by local haemorrhage, which can further damage the brainstem. Studies have shown that patients with basal cistern occlusion, especially in those patients with a significant occlusion, will have significantly lower GCS scores, indicating that their brainstems have primary or secondary damage and are prone to brain herniation.[5] Previous research found that patients with absent or compressed basal cisterns and a midline shift larger than 5 $\mathrm{mm}$ had the highest mortality of $44 \% .[16]$ Therefore, patients with severe basal cistern occlusion are more likely to experience intraoperative acute diffuse brain swelling.

At present, the impact of the timing of surgery on the mortality and functional survival is still controversial. Most previous studies believe that prolonging the timing of surgery may miss the best rescue window, resulting in a poor prognosis, $[5,18,24,25]$ but other clinical scholars have found that neither an operation within 4 hours nor an operation within 6 hours after injury has significant impacts on the mortality; however, with the extension of the timing of surgery, there is increased mortality and a decline in the functional recovery rate.[26] Here, the multivariate logistic regression analysis showed that the time from injury to surgery was an independent risk factor for intraoperative acute diffuse brain swelling. A time from injury to surgery longer than $4 \mathrm{~h}$ was a protective factor against intraoperative acute diffuse brain swelling, suggesting that prolonging the time from injury to surgery can help reduce the risk of intraoperative acute diffuse brain swelling. Kyu-Hong Kim reported that patients who underwent DC within 4 hours had worse outcomes than those patients who underwent surgery at a later time.[27] However, this does not suggest that delaying the operation leads to better outcomes. In our retrospective analysis, the high probability of acute diffuse brain swelling in patients undergoing surgery within 4 hours may be because those patients who seem to be more severely ill and have a lower GCS score will be sent to the advanced neurosurgery trauma centre faster for emergency operative treatment. This selection bias 
will certainly skew the results, and this needs to be verified by more research. In addition, autoregulation of the cerebrovasculature was lost in the patients with bilaterally dilated pupils. Even if we performed a control gradient decompression during the $\mathrm{DC}$, the acute dilation of the cerebrovasculature still cannot be controlled, which ultimately causes intraoperative acute diffuse brain swelling. However, for patients with indications for emergency surgery, there is no doubt that the operations will be performed as soon as possible.

\section{Conclusions}

This study identified the risk factors for intraoperative acute diffuse brain swelling in patients with isolated traumatic ASDH. An increased risk of intraoperative acute diffuse brain swelling occurs in patients with bilaterally dilated pupils, SAH and a short time from injury to surgery. The findings should help neurosurgeons obtain information before surgery about the intraoperative acute diffuse brain swelling in patients with isolated traumatic ASDH. Some limitations of this study should be acknowledged that the retrospective design may lead to bias and uncontrollable confounding factors. For example, early surgery will increase the probability of intraoperative acute diffuse brain swelling, but delayed surgery will often increase the risk of death and disability. These results need to be confirmed by more research in the future.

\section{Abbreviations}

ASDH: acute subdural hematoma; CT: computed tomography; DC: Decompressive craniotomy; GCS: Glasgow Coma Scale; ICP: the intracranial pressure; SAH: subarachnoid haemorrhage; TBI: traumatic brain injury

\section{Declarations}

\section{Ethics approval and consent to participate}

This retrospective study was approved by the Ethics Committee of the Third Affiliated Hospital of Soochow University and all methods were performed in accordance with Declaration of Helsinki. All patients or their authorizers gave written informed consent before the operation.

\section{Consent for publication}

Not applicable.

\section{Availability of data and materials}

All patient data and clinical approaches adopted are contained in the medical files of The Third Affiliated Hospital of Soochow University. The bibliographic data of reference are available on PubMed and the 
conclusions are based on the opinion of the expert involved in this case. The data supporting the conclusions of this article are included within the article and its figures.

\section{Competing interests}

The authors declare that they have no competing interests.

\section{Funding}

This research did not receive any specific grant from funding agencies in the public, commercial, or notfor-profit sectors.

\section{Authors' contributions}

$\mathrm{CL}$ and TM collected patient data. RW performed literature review and drafted the manuscript. HQ and GJ revised the paper and gave the final approval of the definitive version of the article. All authors read and approved the manuscript.

\section{Acknowledgements}

Not applicable.

\section{References}

1. Lv J, Qi X, Wang Y, Wu H, Wang K, Niu H, Li X. Contralateral Subdural Hematoma Following Surgical Evacuation of Acute Subdural Hematoma: Super-Early Intervention and Clinical Implications. World Neurosurg. 2019; 122:24-27.

2. Su TM, Lee TH, Chen WF, Lee TC, Cheng CH. Contralateral acute epidural hematoma after decompressive surgery of acute subdural hematoma: clinical features and outcome. $J$ Trauma. 2008; 65(6):1298-1302.

3. Shen J, Pan JW, Fan ZX, Zhou YQ, Chen Z, Zhan RY. Surgery for contralateral acute epidural hematoma following acute subdural hematoma evacuation: five new cases and a short literature review. Acta Neurochir (Wien). 2013; 155(2):335-341.

4. Dent DL, Croce MA, Menke PG, Young BH, Hinson MS, Kudsk KA, Minard G, Pritchard FE, Robertson JT, Fabian TC. Prognostic factors after acute subdural hematoma. J Trauma. 1995; 39(1):36-42; discussion 42-33.

5. Wilberger JE, Walters BC, Servadei F, Newell DW, Hartl R, Gordon D, Ghajar J, Chesnut R, Bullock MR. Surgical Management of Acute Subdural Hematomas. Neurosurgery. 2006; 58(suppl_3):S2-16-S1224.

6. Li LM, Kolias AG, Guilfoyle MR, Timofeev I, Corteen EA, Pickard JD, Menon DK, Kirkpatrick PJ, Hutchinson PJ. Outcome following evacuation of acute subdural haematomas: a comparison of craniotomy with decompressive craniectomy. Acta Neurochir (Wien). 2012; 154(9):1555-1561. 
7. Leitgeb J, Mauritz W, Brazinova A, Janciak I, Majdan M, Wilbacher I, Rusnak M. Outcome after severe brain trauma due to acute subdural hematoma. J Neurosurg. 2012; 117(2):324-333.

8. Woertgen C, Rothoerl RD, Schebesch KM, Albert R. Comparison of craniotomy and craniectomy in patients with acute subdural haematoma. J Clin Neurosci. 2006; 13(7):718-721.

9. Gumussoy M, Ugur O, Cukurova I, Uluyol S. Recurrent meningitis and frontal encephalocele as delayed complications of craniofacial trauma. J Craniofac Surg. 2014; 25(2):529-530.

10. Chauny JM, Marquis M, Bernard F, Williamson D, Albert M, Laroche M, Daoust R. Risk of Delayed Intracranial Hemorrhage in Anticoagulated Patients with Mild Traumatic Brain Injury: Systematic Review and Meta-Analysis. J Emerg Med. 2016; 51(5):519-528.

11. Jungner M, Lundblad C, Bentzer P. Rosuvastatin in experimental brain trauma: improved capillary patency but no effect on edema or cerebral blood flow. Microvasc Res. 2013; 88:48-55.

12. Rosenblatt AJ, Scrivani PV, Caserto BG, Ruby RE, Loftus JP, de Lahunta A, Noden DM. Imaging diagnosis-meningoencephalitis secondary to suppurative rhinitis and meningoencephalocele infection in a dog. Vet Radiol Ultrasound. 2014; 55(6):614-619.

13. Sahuquillo J, Poca MA, Amoros S. Current aspects of pathophysiology and cell dysfunction after severe head injury. Curr Pharm Des. 2001; 7(15):1475-1503.

14. Xu F-h, Chen W-j, Yang Y-j, Duan Y-x, Fu F-I. Evaluation of multislice computed tomographic perfusion imaging and computed tomographic angiography on traumatic cerebral infarction. Chinese Journal of Traumatology (English Edition). 2008; 11(3):186-189.

15. Taussky P, Widmer HR, Takala J, Fandino J. Outcome after acute traumatic subdural and epidural haematoma in Switzerland: a single-centre experience. Swiss Med Wkly. 2008; 138(19-20):281-285.

16. Maas Al, Hukkelhoven CW, Marshall LF, Steyerberg EW. Prediction of outcome in traumatic brain injury with computed tomographic characteristics: a comparison between the computed tomographic classification and combinations of computed tomographic predictors. Neurosurgery. 2005; 57(6):1173-1182; discussion 1173-1182.

17. Jamous M, Barbarawi M, Samrah S, Khabaz MN, Al-Jarrah M, Dauod S. Emergency decompressive craniectomy for trauma patients with Glasgow Coma Scale of 3 and bilateral fixed dilated pupils. Eur J Trauma Emerg Surg. 2010; 36(5):465-469.

18. Tian HL, Chen SW, Xu T, Hu J, Rong BY, Wang G, Gao WW, Chen H. Risk factors related to hospital mortality in patients with isolated traumatic acute subdural haematoma: analysis of 308 patients undergone surgery. Chin Med J (Engl). 2008; 121(12):1080-1084.

19. Wardlaw JM, Easton VJ, Statham P. Which CT features help predict outcome after head injury? J Neurol Neurosurg Psychiatry. 2002; 72(2):188-192; discussion 151.

20. Yoshikawa G, Kawamoto S, Ono H, Yakou K, Shinozaki M, Tsutsumi K. [Vertebrobasilar artery dissection with subarachnoid hemorrhage after brain stem infarct showing an improvement on angiography: case report]. No Shinkei Geka. 2003; 31(7):803-809.

21. Servadei F, Murray GD, Teasdale GM, Dearden M, lannotti F, Lapierre F, Maas AJ, Karimi A, Ohman J, Persson L et al. Traumatic subarachnoid hemorrhage: demographic and clinical study of 750 
patients from the European brain injury consortium survey of head injuries. Neurosurgery. 2002; 50(2):261-267; discussion 267-269.

22. Ono J, Yamaura A, Kubota $\mathrm{M}$, Okimura $\mathrm{Y}$, Isobe $\mathrm{K}$. Outcome prediction in severe head injury: analyses of clinical prognostic factors. J Clin Neurosci. 2001; 8(2):120-123.

23. Servadei F, Nasi MT, Giuliani G, Cremonini AM, Cenni P, Zappi D, Taylor GS. CT prognostic factors in acute subdural haematomas: the value of the 'worst' CT scan. Br J Neurosurg. 2000; 14(2):110-116.

24. Leach P, Childs C, Evans J, Johnston N, Protheroe R, King A. Transfer times for patients with extradural and subdural haematomas to neurosurgery in Greater Manchester. Br J Neurosurg. 2007; 21(1):11-15.

25. Mondello S, Shear DA, Bramlett HM, Dixon CE, Schmid KE, Dietrich WD, Wang KK, Hayes RL, Glushakova O, Catania M et al. Insight into Pre-Clinical Models of Traumatic Brain Injury Using Circulating Brain Damage Biomarkers: Operation Brain Trauma Therapy. J Neurotrauma. 2016; 33(6):595-605.

26. Zhao H, Bai XJ. Influence of operative timing on prognosis of patients with acute subdural hematoma. Chin J Traumatol. 2009; 12(5):296-298.

27. Kim KH. Predictors for functional recovery and mortality of surgically treated traumatic acute subdural hematomas in 256 patients. J Korean Neurosurg Soc. 2009; 45(3):143-150.

\section{Figures}




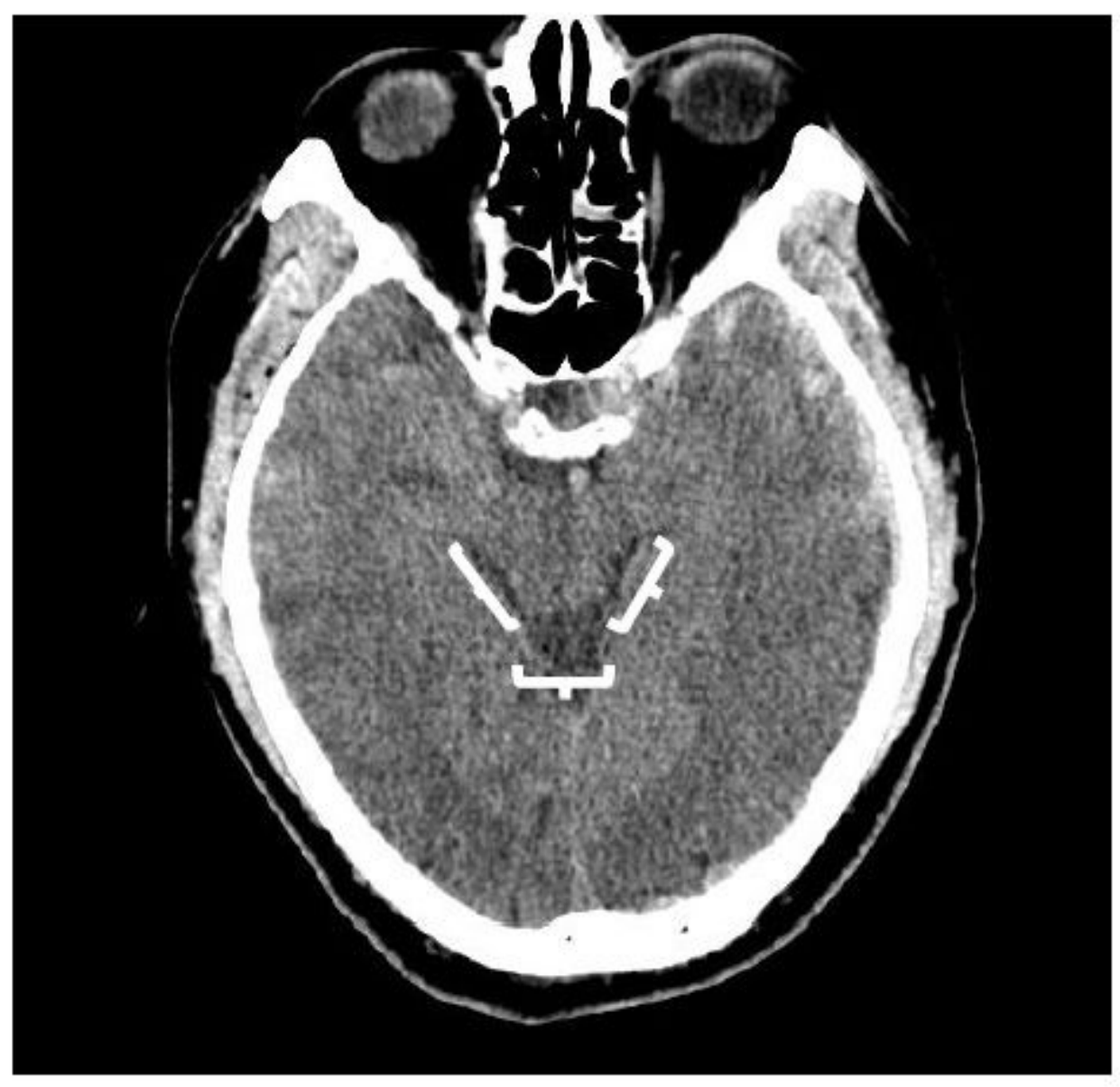

Figure 1

Evaluation of the basal cisterns on computed tomographic scan. 